Applied nutritional investigation

\title{
The metabolite beta-aminoisobutyric acid and physical inactivity among hemodialysis patients
}

\author{
Alessio Molfino M.D., Ph.D. ${ }^{\text {a, }}$, Maria Ida Amabile M.D., Ph.D. ${ }^{a}$,

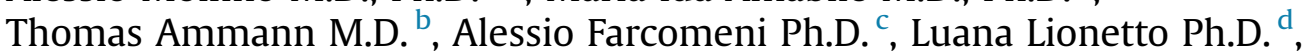 \\ Maurizio Simmaco M.D. ${ }^{e}$, Silvia Lai M.D. ${ }^{a}$, Alessandro Laviano M.D. ${ }^{a}$, \\ Filippo Rossi Fanelli M.D. ${ }^{\mathrm{a}}$, Maria Grazia Chiappini M.D. ${ }^{\mathrm{b}}$, \\ Maurizio Muscaritoli M.D. ${ }^{\text {a }}$ \\ a Department of Clinical Medicine, Sapienza University of Rome, Rome, Italy \\ b Hemodialysis Unit, Fatebenefratelli 'Isola Tiberina' Hospital, Rome, Italy \\ ${ }^{\mathrm{c}}$ Department of Public Health and Infectious Diseases, Sapienza University of Rome, Rome, Italy \\ ${ }^{\mathrm{d}}$ Personalized Medicine Unit, Istituto Dermopatico dell'Immacolata-IRCCS, Rome, Italy \\ e Neuroscience, Mental Health and Sense Organs Department, Advanced Molecular Diagnostics Unit, Sant'Andrea Hospital, Rome, Italy
}

\section{A R T I C L E I N F O}

Article history:

Received 21 February 2016

Accepted 19 July 2016

\section{Keywords:}

Beta-aminoisobutyric acid (BAIBA)

Physical activity

Hemodialysis

Body composition

\begin{abstract}
A B S T R A C T
Objective: Physical inactivity is frequent in patients on hemodialysis (HD), and represents a reliable predictor of morbidity and mortality. Beta-aminoisobutyric acid (BAIBA) is a contraction-induced myokine, the plasma levels of which increase with exercise and are inversely associated with metabolic risk factors. The aim of this study was to ascertain whether physical inactivity and clinical parameters relate to plasma BAIBA levels in this patient population.

Methods: Adult patients on HD were included, and the presence of physical inactivity was assessed. BAIBA levels were measured in these patients and in healthy individuals. We assessed barriers to physical activity, including 23 items regarding psychophysical and financial barriers. Body composition was assessed by bioimpedance and muscle strength by handgrip dynamometer. Nonparametric tests and logistic regression analyses were performed.

Results: Forty-nine patients on HD were studied; $49 \%$ were physically active and $51 \%$ were inactive. Of the patients, 43 reported barriers to physical activity and $61 \%$ of inactive patients reported three or more barriers. BAIBA levels were lower in patients on HD with respect to controls $(P<0.001)$. Stratifying HD patients as active and inactive, both groups showed significantly lower BAIBA levels versus controls $(P=0.0005, P<0.001$, respectively). Nondiabetic patients on HD showed increased BAIBA levels compared with diabetic patients $(P<0.001)$. Patients on HD endorsing the two most frequent barriers showed lower BAIBA levels than those not reporting these barriers $(P=0.006)$. Active patients showed higher intracellular water $(\%)(P=0.008)$, and active and inactive patients showed significant correlation between total body muscle mass and handgrip strength $(P=0.04$, $P=0.005$, respectively).

Conclusions: Physical inactivity is highly prevalent among patients on HD and BAIBA correlates with barriers to physical activity reported by inactive patients.
\end{abstract}

(c) 2016 Elsevier Inc. All rights reserved.
This study was supported by Institutional funds (Department of Clinical
Medicine, Sapienza - University of Rome). AM designed and conducted the
research, analyzed the data, and wrote the paper. MIA conducted research,
collected the data, and wrote the paper. TA collected the data. AF analyzed data
and performed statistical analyses. LL performed laboratory dosage. MS analyzed
laboratory data. SL conducted research. AL designed research and reviewed the
paper. FRF designed research. MGC conducted research, enrolled the patients, and reviewed the paper. MM designed research, reviewed the paper, and had primary responsibility for final content.

All authors read and approved the final manuscript. The authors have no conflicts of interest to declare.

* Corresponding author. Tel./fax: +39064 9972042.

E-mail address: alessio.molfino@uniroma1.it (A. Molfino). 


\section{Introduction}

Physical activity is significantly reduced in patients on hemodialysis (HD) [1]. Independently from age, a low level of physical activity may significantly contribute to frailty and disability by reducing physical performance and may determine protein-energy wasting, which is highly prevalent among this patient population [2], by inducing skeletal muscle atrophy [1]. Low levels of physical activity have been associated with reduced survival in dialysis patients and physical inactivity is a strong predictor of cardiovascular mortality in HD [3]. The presence of several comorbidities, in association with socioeconomic, psychological, and cultural inabilities, plays a key role in conditioning physical inactivity [4].

Although physical activity can be easily investigated using quantitative tools, most physicians do not assess physical activity or inactivity in patients on HD [5], and the choice of instrument to be used depends on the patient's activity level and on the outcomes of interest. In particular, most of the questionnaires used were not specifically designed to be implemented in dialysis patients.

To our knowledge, there is no specific biomarker by which to diagnose the reduced level of physical activity in dialysis patients, which could be useful for diagnosis and during follow-up visits and would not be time consuming.

Recently, beta-aminoisobutyric acid (BAIBA) has been identified as a contraction-induced myokine [6]. BAIBA is a nonprotein beta-amino acid generated by the catabolism of the branchedchain amino acid valine. Skeletal muscle is a major site of branched-chain amino acid utilization, the catabolism of which is significantly increased during exercise $[7,8]$. The forced expression of the muscle peroxisome proliferator-activated receptor- $\gamma$ coactivator-1 $\alpha$ regulates BAIBA expression during exercise [6]. Available data suggest a possible link between valine use in skeletal muscle during exercise and beneficial effects of BAIBA in inducing browning of white fat and hepatic $\beta$-oxidation through a peroxisome proliferator-activated receptor- $\alpha$-mediated mechanism [5]. Thus, we hypothesized that BAIBA levels may be significantly reduced in patients with reduced physical activity. The primary aim of this study was to assess the prevalence of physical inactivity and its relationship with BAIBA levels as a possible novel biomarker of physical activity in a cohort of patients on HD.

The secondary aim was to relate BAIBA levels with metabolic and inflammatory alterations, body composition modifications, and low muscle strength.

\section{Material and methods}

This cross-sectional study was performed on patients from the Dialysis Unit of "Fatebenefratelli" Hospital, Isola Tiberina, in Rome, Italy. Adult men and women on HD who were participants of a study investigating the role of nutrition and quality of life were enrolled. The study was approved by the local ethics committee (ethics committee registration n. 29/2013). Written informed consent was obtained from all patients before enrollment in the study. More than 50 patients with end-stage renal disease were being treated in this hemodialysis unit. All adult patients receiving HD in October 2014 were recruited for this study. Exclusion criteria included presence of highly catabolic diseases, such as cancer, chronic infections, and AIDS, and the absence of informed consent. Patients were maintained on a regular HD prescription, three times a week, for 4-h sessions.

\section{Patient demographic and clinical characteristics}

Participants were interviewed before or during dialysis session, and demographic characteristics, including age, sex, weight, height, body mass index (BMI), dialysis vintage, and $\mathrm{Kt} / \mathrm{V}$ were registered. Hemoglobin and serum nutritional and inflammatory biomarkers-including total proteins, cholesterol, triglycerides, albumin, normalized protein catabolic rate, C-reactive protein, lymphocytes, transferrin, blood urea nitrogen, sodium, potassium, phosphorus, and creatinine-were also recorded. Additionally, comorbidities, blood pressure control, and current therapies were collected. C-reactive protein was measured by nephelometric assay (detection limit $=0.5 \mathrm{mg} / \mathrm{dL}$ ).

\section{Body impedance analysis}

Body impedance analysis was used to assess body composition, in particular to evaluate muscle mass and adiposity [9], and was performed $1 \mathrm{~h}$ after the end of the second HD session of the week, with the patient maintaining a supine position for this period, using a single-frequency $(50 \mathrm{~Hz})$ instrument (Model 101, Akern, Florence, Italy). Data previously obtained by body impedance analysis as soon as $15 \mathrm{~min}$ after starting the dialysis session has been predictable [9]. The electrodes and recording pads were placed on the nonaccess side of the body. Two pairs of electrodes were placed on the hand to the foot for injecting current and on the wrist to the ankle for measuring voltage. Total body water was estimated using the resistance extrapolated to frequency [9]. We estimated the hydration of fat free mass (FFM) at $73 \%$ (FFM $=$ [total body water] $/ 0.73$ ) and extracellular water was estimated from resistance extrapolated to zero frequency [9-11]. Additional parameters, including body cell mass, were calculated using a program provided by the producer. We calculated fat mass as the difference between body weight and FFM and FFM index was calculated as FFM (kg)/height $(\mathrm{m})^{2}$. Total body muscle mass was calculated as previously described [12].

\section{Muscle strength}

Muscle function was assessed by handgrip strength measurement on the same day of the recruitment before starting the HD session. A dynamometer handle (JAMAR, Sammons Preston Rolyan, Bolingbrook, IL, USA) was used with the purpose of measuring the maximum isometric strength of the hand and forearm muscles. The test was conducted by performing three attempts with each hand, and the mean of the strongest hand was used to determine muscle strength [13]. The strength measurement was adjusted by sex and BMI.

\section{Physical activity level}

Two simple questions were administered to patients enrolled in the present study. The first question concerned the self-reported frequency of physical activity, including running, recreational walking, and so on, during leisure time. Six answer options were available according to physical activity level: daily or almost daily, four to five times a week, 2 to 3 times a week, once a week, less than once a week, and almost never or never, as previously described by Tentori et al. [14]. Patients were divided into two groups: those who never exercised or reported physical exercise less than once a week (inactive), and those who experienced exercise more often (active) [14]. The second question regarded barriers to physical activity in general or to lower physical activity levels than desired. It consisted of investigating 23 barriers, as shown in Table 1 [15], designed on the basis of previously validated questions [16].

Additionally, we asked patients to answer the Activities of Daily Living (ADL) score questionnaire, consisting of six questions [17]. The maximum obtainable score was 6 . Participants reporting dependency in one or more daily activity were considered to have an ADL limitation [17].

\section{BAIBA assay}

Blood samples were obtained from HD patients immediately before the second HD session of the week. We also collected blood samples from 43 healthy, active individuals (control group). Blood $(5 \mathrm{~mL}$ ) was sampled in anticoagulantfree tubes and kept at room temperature for $1 \mathrm{~h}$ before the serum was isolated. Aliquots of serum were stored at $-80^{\circ} \mathrm{C}$ until analysis. BAIBA levels $(\mu \mathrm{M})$ were blind measured at the Neuroscience, Mental Health and Sense Organs Department, Sapienza University of Rome, Rome, Italy. Standards of D,L-3-aminoisobutyric acid and internal standard norvaline were used (Sigma Aldrich, Saint Louis, MO, USA). High-performance liquid chromatography-grade acetonitrile (Carlo Erba, Milan, Italy) and formic acid (Merck, Darmstadt, Germany) were also used. Water was deionized and filtered with a Milli-Q Plus apparatus (Millipore Corporation, Bedford, MA, USA).

Forty $\mu \mathrm{L}$ of serum samples were added to $160 \mu \mathrm{L}$ of internal standard in acetonitrile. The samples were vortex-mixed for $10 \mathrm{~s}$ and centrifuged at $14000 \mathrm{~g}$ for $5 \mathrm{~min}$ and $100 \mu \mathrm{L}$ of clean upper laver were diluted with $100 \mu \mathrm{L}$ of $0.1 \%$ formic acid and transferred in autosampler vials. Thirty $\mu \mathrm{L}$ were injected into the chromatographic system. The high-performance liquid chromatography analysis was performed using an Agilent Liquid Chromatography System series 1100 (Agilent Technologies, Santa Clara, CA, USA) including a binary pump, an autosampler, a solvent degasser, and a column oven. The column was a Phenomenex Luna HILIC $(100 \times 2.0 \mathrm{~mm}, 3 \mu \mathrm{m}$; Phenomenex, Torrance, CA, USA $)$ equipped with a security-guard precolumn (Phenomenex) containing the same packing material. The column was maintained at room temperature. The mobile phase consisted of a solution of $0.1 \%$ aqueous formic acid, $10 \mathrm{mM}$ ammonium formate 
Table 1

Patient-reported barriers to physical activity

\begin{tabular}{|c|c|c|c|}
\hline \multirow[t]{2}{*}{ Reported barriers } & \multicolumn{2}{|c|}{ Total participants $(\mathrm{N}=49)$} & \multirow[t]{2}{*}{$P$ value } \\
\hline & $\begin{array}{l}\text { Inactive } \\
\text { participants } \\
(\mathrm{n}=25)\end{array}$ & $\begin{array}{l}\text { Active } \\
\text { participants } \\
(\mathrm{n}=24)\end{array}$ & \\
\hline 1 Fatigue on the dialysis days & 24 & 13 & $<0.001$ \\
\hline 2 Reduced walking ability & 15 & 4 & $<0.001$ \\
\hline 3 Muscle pain & 4 & 0 & 0.05 \\
\hline 4 Pain during walking & 7 & 3 & 0.17 \\
\hline 5 Too many medical problems & 10 & 1 & 0.002 \\
\hline 6 Leg pain & 7 & 1 & 0.002 \\
\hline 7 Fear of getting hurt & 6 & 0 & 0.01 \\
\hline 8 Hip pain & 8 & 2 & 0.04 \\
\hline 9 "I don't want to" & 13 & 5 & 0.02 \\
\hline $\begin{array}{l}10 \text { Fatigue on the nondialysis } \\
\text { days }\end{array}$ & 10 & 2 & 0.008 \\
\hline 11 Shortness of breath & 14 & 7 & 0.04 \\
\hline 12 Feet pain & 4 & 1 & 0.19 \\
\hline 13 Mood disturbances & 1 & 0 & 0.49 \\
\hline 14 Sadness & 2 & 1 & 0.61 \\
\hline 15 Anxiety & 2 & 0 & 0.23 \\
\hline 16 No exercise partner & 1 & 0 & 0.49 \\
\hline 17 No place to exercise & 0 & 0 & $>0.99$ \\
\hline 18 Heart disease & 4 & 2 & 0.42 \\
\hline 19 Feeling too old & 11 & 3 & 0.01 \\
\hline 20 Ulcers on legs and feet & 2 & 1 & 0.61 \\
\hline 21 No one suggested me to exercise & 2 & 1 & 0.61 \\
\hline 22 "I have no time" & 1 & 3 & 0.61 \\
\hline 23 Chest pain & 1 & 0 & 0.49 \\
\hline
\end{tabular}

Bold values indicate statistically significant $P$ values.

(eluent A), and $0.1 \%$ formic acid in acetonitrile (eluent B): Elution was performed at flow rate of $300 \mu \mathrm{L} / \mathrm{min}$, using an elution gradient. The injection volume was $30 \mu \mathrm{L}$, and the total analysis run time was $15 \mathrm{~min}$. The mass spectrometry method was performed on a 3200 triple quadrupole system (Applied Biosystems, Foster City, CA, USA) equipped with a turbo ion spray source. The detector was set in positive ion mode. The ion spray voltage was set at $5000 \mathrm{~V}$ and the source temperature was $400^{\circ} \mathrm{C}$, the collision activation dissociation gas was set at medium value, and nitrogen was used as collision gas. The Q1 and Q3 quadrupoles were tuned for the unit mass resolution. The instrument was set in the multiple reaction monitoring mode. The specific ion $(\mathrm{m} / \mathrm{z})$ monitored was BAIBA $(\mathrm{m} / \mathrm{z}$ $104.1 \rightarrow \mathrm{m} / \mathrm{z}$ 87.0) with a dwell time of $300 \mathrm{~ms}$. Mass spectrometer parameters were optimized to maximize sensitivity. Data were acquired and processed by Analyst 1.5.1 Software.

\section{Statistical analyses}

Patient characteristics were described using mean \pm SD for continuous normally distributed variables and percent for dichotomous variables. Variables that were not normally distributed were described using median with 25 th and 75th percentiles. The proportion of patients self-reporting each barrier and the average number of barriers perceived per patient were calculated. Chi-square, Mann-Whitney, and Kruskall-Wallis tests were used to study the association between reported barriers to physical activity and patient characteristics, the association between barriers and reported levels of physical activity, and between BAIBA and levels of physical activity. Spearman's correlation index was used to assess associations between continuous measurements. Univariate logistic regression was used to evaluate odds ratios of factors associated with binary outcomes (e.g., active/inactive).

Multivariable regression and logistic regression analysis was used for the same aims, adjusting for other patient characteristics often associated with inactivity (e.g., age, sex, comorbidity). The best multivariable model was selected by minimizing the Akaike information criterion. Physical activity was treated as a categorical variable for these analyses. A standard two-tailed $\alpha(P<0.05)$ was considered statistically significant. All statistical analysis was performed in $\mathrm{R} v$. 3.0.2.

\section{Results}

\section{Patient's characteristics}

Fifty-two patients on HD were enrolled in the study. Three participants were excluded: One was transferred to another dialysis center shortly after the beginning of the study, and two were excluded because they did not perform bioimpedance analysis. Therefore, 49 patients were studied (36 men and 13 women) with a mean age of $66.6 \pm 15.5 \mathrm{y}$. Median dialysis vintage was 37 mo (interquartile range [IQR], 13-92) with a mean $\mathrm{Kt} / \mathrm{V}$ of $1.3 \pm 0.3$. Fifteen patients were treated with highpermeability membranes and 34 patients with low-permeability membranes.

Characteristics of the enrolled patients are reported in Table 2. The dialytic diagnosis was chronic renal failure due to unknown or uncertain etiology in 15 patients, diabetic nephropathy in 8 patients, vascular diseases in 7 patients, chronic hereditary or unknown origin glomerulonephritis in 7 patients, and other diagnosis in 12 patients. The mean normalized protein catabolic rate was $0.97 \pm 0.22 \mathrm{~g} / \mathrm{kg}$ daily.

Of the enrolled patients, $22 \%$ presented two or more comorbidities, $71 \%$ suffered from arterial hypertension, $30 \%$ had hyperlipidemia, and $26 \%$ had diabetes. The majority of the participants were treated with antihypertensive therapies, erythropoietin, statins, potassium and phosphate binders, and vitamin supplements. All patients were supplemented with folic acid.

There were 43 control participants ( 21 men, 22 women) with a mean age of $66.7 \pm 1.1 \mathrm{y}$ and a normal BMI $\left(18.5-24.9 \mathrm{~kg} / \mathrm{m}^{2}\right)$.

\section{Self-reported physical activity}

The enrolled patients were generally sedentary, as 51\% reported engaging in physical activity during leisure time less than once per week, almost never, or never. We identified this group as inactive. Only $26 \%$ of patients, identified as active, reported doing physical activity during leisure time daily or almost daily (four to five times a week). Forty-three patients acknowledged having barriers to physical activity. Among them, 61\% reported having at least three barriers to physical activity. Only $6 \%$ of the enrolled patients did not report any barriers to physical activity. The most self-reported barrier was "fatigue on the dialysis days" (73\%), followed by "shortness of breath" (40\%), "reduced walking ability" (38\%), "I don't want to" (38\%), and "heart disease" (36\%). "Feeling too old" as a barrier to physical activity was reported by $28 \%$ of patients. Only $8 \%$ patients reported muscle pain as a

Table 2

Patient characteristics $(\mathrm{N}=49)$

\begin{tabular}{lc}
\hline Characteristics & Mean $\pm \mathrm{SD}^{*}$ \\
\hline Sex (male:female) & $36: 13$ \\
Age, y & $66.6 \pm 15.5$ \\
Dialysis vintage, mo & $37(13-92)$ \\
Body weight, kg & $69.4 \pm 16.5$ \\
BMI, kg/m ${ }^{2}$ & $24.8 \pm 5.5$ \\
nPCR, g/kg/d & $0.9 \pm 0.2$ \\
Creatinine, mg/dL & $9.4 \pm 2.5$ \\
Urea, mg/dL & $129.4 \pm 27$ \\
Kt/V & $1.3 \pm 0.3$ \\
Albumin, g/dL & $36.7 \pm 2.8$ \\
Lymphocytes, n/mm ${ }^{3}$ & $1.6 \pm 0.6$ \\
CRP, mg/dL & $0.40(0.11-0.81)$ \\
Cholesterol, mg/dL & $146.5 \pm 34.9$ \\
Hb, g/dL & $11.4 \pm 1.4$ \\
Comorbidities, yes/no & \\
Hypertension & $35 / 14$ \\
Diabetes mellitus & $13 / 36$ \\
Hyperlipidemia & $16 / 33$ \\
\hline
\end{tabular}

BMI, body mass index; CRP, C-reactive protein; Hb, hemoglobin; nPCR, normalized protein catabolic rate; SD, standard deviation

* Median (interquartile range) is shown for nonnormally distributed variable (dialysis vintage; C-reactive protein). 
barrier. ADL score was 6 out of 6 for $81 \%$ of patients. All participants in the control group were active according to this questionnaire.

\section{BAIBA, physical activity, and clinical parameters}

BAIBA $(\mu \mathrm{M})$ median value in all HD patients was 0.40 (IQR, 0.21-0.61), whereas in controls it was 0.98 (IQR, 0.63-1.66; Fig. 1).

In univariate analysis, BAIBA levels were significantly lower in HD patients than in healthy controls $(P<0.001$; Fig. 1$)$. Stratifying HD patients as active and inactive, both groups showed significantly lower BAIBA levels than control $(P=0.0005$ and $P<0.001$, respectively; Fig. 2). BAIBA levels were lower in inactive HD patients than in active HD patients, although not statistically significant ( $P=0.09$; Fig. 2 ).

No correlation was found among BAIBA levels, BMI, and body composition parameters or with serum biomarkers, including inflammatory status, with the exception of high-density lipoprotein cholesterol $(r=0.40 ; P=0.004)$, independently from the level of physical activity. The type of dialysis (high or low permeability membranes) did not influence BAIBA levels.

Among active and inactive patients, BAIBA levels did not correlate with the number of comorbidities, but, after excluding patients with a short dialysis vintage $(\leq 3 \mathrm{mo})$, this correlation was present $(r=-0.31 ; P=0.04)$. When considering the type of comorbidities, patients without diabetes displayed higher median BAIBA levels than those with the disease $(0.43 \mu \mathrm{M}$, IQR $0.26-0.85$ versus $0.32 \mu \mathrm{M}$, IQR $0.14-0.43 ; P<0.001)$ in both groups of patients. No correlation was found between BAIBA levels and hypercholesterolemia and/or statin treatment.

BAIBA levels correlated with the two most frequent barriers reported by inactive patients (Table 1 ). In particular, "fatigue on the dialysis days" correlated with BAIBA-circulating levels $(P=0.05)$. Also, inactive patients endorsing both "fatigue on the dialysis days" and "reduced walking ability" showed lower median BAIBA levels $(\mu \mathrm{M})$ versus HD patients not reporting these two barriers, independently from the activity level $(0.26 \mu \mathrm{M}, 95 \%$

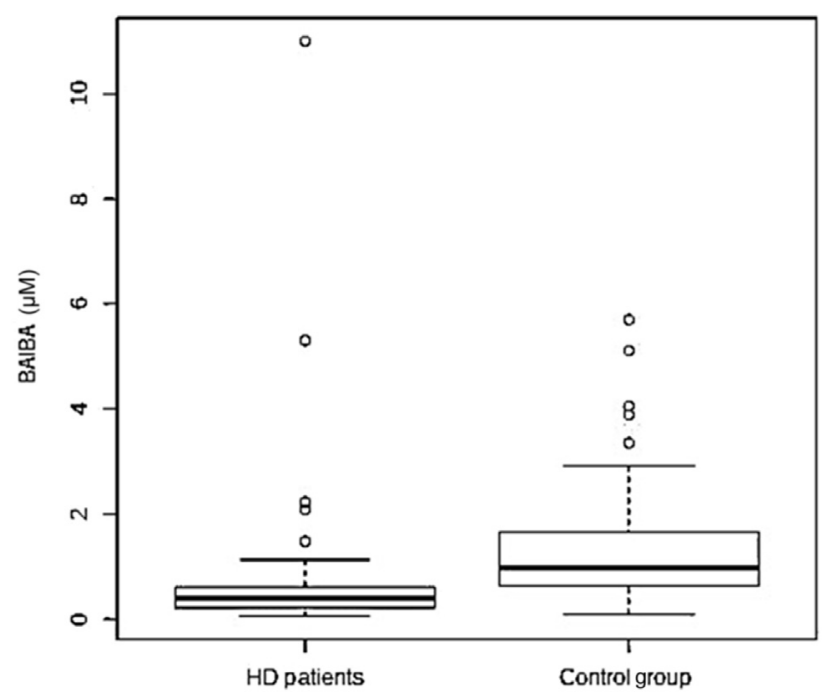

Fig. 1. BAIBA plasma levels in hemodialysis patients and in controls. Box plot of BAIBA levels $(\mu \mathrm{M})$ stratified by patients on HD or control group. BAIBA levels were significantly lower in HD patients respect to control group $(P<0.001)$. Lines represent the median, 25th, and 75th percentiles, and the whiskers (error bars) below and above the box indicate the 10th and 90th percentiles. BAIBA, betaaminoisobutyric acid; HD, hemodialysis.

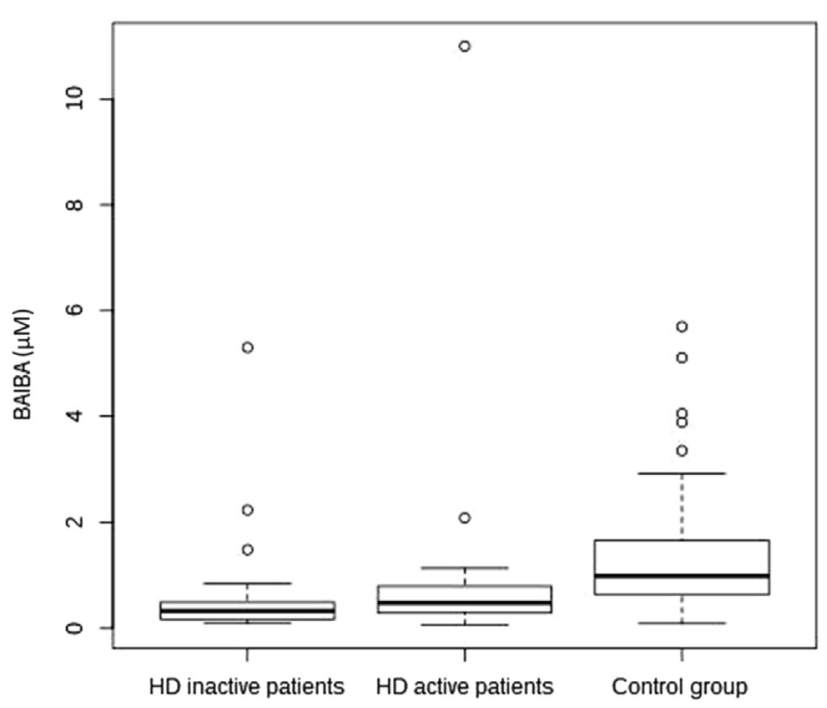

Fig. 2. BAIBA plasma levels in active and inactive hemodialysis patients, and in controls. Box plot of BAIBA levels $(\mu \mathrm{M})$ stratified by control group or "active" or "inactive" patients on HD. Both "active" and "inactive" groups showed significantly lower BAIBA levels versus control $(P=0.0005$ and $P<0.001$, respectively). Lines represent the median, 25th, and 75th percentiles, and the whiskers (error bars) below and above the box indicate the 10th and 90th percentiles. BAIBA, betaaminoisobutyric acid; HD, hemodialysis.

confidence interval [CI] 0.12-0.43 versus $0.47 \mu \mathrm{M}, 95 \% \mathrm{CI} 0.29$ 0.85 , respectively; $P=0.006$; Fig. 3 ). No correlation between BAIBA and handgrip strength measures has been documented.

\section{Association between barriers and self-reported physical activity}

In univariate analysis, "fatigue on the dialysis days" and "reduced walking ability" were associated with inactivity

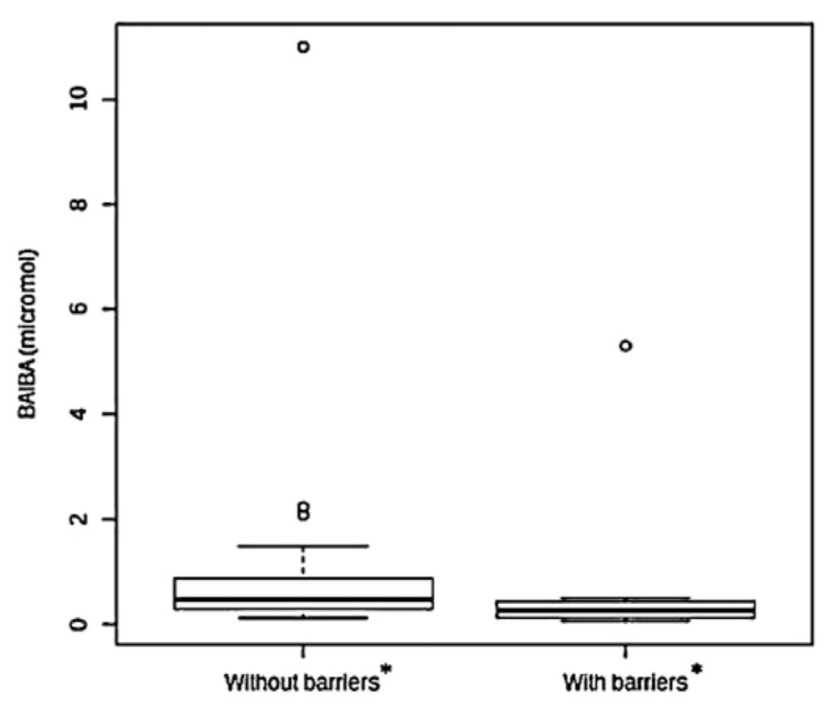

Fig. 3. BAIBA plasma levels and barriers to physical activity. Box plot of BAIBA levels $(\mu \mathrm{M})$ stratified by HD patients endorsing barriers "fatigue on the dialysis days" and "reduced walking ability" (the two most frequent barriers in inactive patients) or HD patients not self-reporting these two barriers. HD patients with the two barriers had lower BAIBA levels $(\mu \mathrm{M})$ than those patients without the two barriers, independently from the activity level $(0.26 \mu \mathrm{M}, 95 \% \mathrm{CI}, 0.12-0.43$ versus $0.47 \mu \mathrm{M}, 95 \% \mathrm{CI}$, $0.29-0.85$, respectively; $P=0.006$ ). Lines represent the median, 25 th, and 75 th percentiles, and the whiskers (error bars) below and above the box indicate the 10th and 90th percentiles. BAIBA, beta-aminoisobutyric acid; HD, hemodialysis. *"Fatigue on the dialysis days" and "reduced walking ability." 
$(P=0.0003$ and $P=0.0007$, respectively), similarly to "fatigue on the non-dialysis days" $(P=0.008$; Table 1$)$. Patients who endorsed "ulcers on legs and feet" or "feet pain" as a barrier were not less likely to be active ( $P=0.68$ and $P=0.19$, respectively). Hip and leg pain were barriers associated with inactivity ( $P=0.04$ and $P=0.002$, respectively). Reporting "too many medical problems" or "fear of getting hurt" was associated with inactivity $(P=0.002$ and $P=0.01$, respectively). "Feeling too old" or "I don't want to perform physical activity" were barriers associated with inactivity ( $P=0.01$ and $P=0.02$, respectively). None of the participants endorsed any "lack of a location to exercise" as a barrier to physical activity. A significant correlation between the number of barriers reported by each participant and the number of comorbidities was detected $(r=0.29 ; P=0.04)$. No differences in blood pressure control and in other comorbidities, including diabetes or the presence of anemia, low Kt/v, and low albumin-which are generally associated with asthenia-were found between active and inactive patients. In multivariate analysis, after adjustment for age and sex, only the number of barriers reported by each patient resulted in significant associations with physical inactivity (odds ratio [OR], 4.22; 95\% CI, 1.72-14.00; $P=0.006)$.

Body composition, electrolytes, handgrip, and self-reported physical activity

Body composition parameters between active and inactive patients are reported in Table 3. In particular, active patients showed higher intracellular water $(\%)(P=0.008)$, cellular mass (\%) $(P=0.005)$, and body mass cell index $(P=0.04)$. Serum electrolytes, including sodium, did not differ between the two groups (Table 3 ).

Handgrip strength $(\mathrm{kg})$ was $24.5 \pm 9.7$. No differences in handgrip values between active and inactive patients were observed ( $P=0.07$; Table 3 ), however, there was a robust correlation between total body muscle mass and handgrip strength when considering all HD patients $(r=0.55 ; P<0.0001)$. Both

Table 3

Body composition parameters, electrolytes, and functional assessment of all patients $(\mathrm{N}=49)$

\begin{tabular}{|c|c|c|c|}
\hline Parameters & $\begin{array}{l}\text { Active } \\
\text { patients } \\
(n=24)\end{array}$ & $\begin{array}{l}\text { Inactive } \\
\text { patients } \\
(\mathrm{n}=25)\end{array}$ & $P$ value \\
\hline Body weight, kg & $69.9 \pm 18.4$ & $69.2 \pm 14.7$ & 0.88 \\
\hline \multicolumn{4}{|l|}{ Body composition parameter } \\
\hline FFM, $\%$ & $66.8 \pm 8.9$ & $68.4 \pm 8.0$ & 0.52 \\
\hline FFM, kg & $46.5 \pm 13$ & $47.2 \pm 11.2$ & 0.83 \\
\hline FFM index, $\mathrm{kg} / \mathrm{m}^{2}$ & $17.0 \pm 4.1$ & $17.0 \pm 3.1$ & $>0.99$ \\
\hline Fat mass, $\%$ & $33.2 \pm 8.9$ & $31.6 \pm 8.0$ & 0.52 \\
\hline Fat mass, kg & $23.3 \pm 9.2$ & $21.0 \pm 7.2$ & 0.58 \\
\hline Intracellular water, liter & $18.2 \pm 5.7$ & $16.1 \pm 4.9$ & 0.17 \\
\hline Intracellular water, \% & $51.2 \pm 7.3$ & $44.5 \pm 9.5$ & 0.008 \\
\hline Extracellular water, liter & $17.5 \pm 5.6$ & $20.3 \pm 5.9$ & 0.1 \\
\hline Extracellular water, \% & $48.8 \pm 7.3$ & $55.3 \pm 9.6$ & 0.01 \\
\hline Body mass cell index, $\mathrm{kg} / \mathrm{m}^{2}$ & $6.7 \pm 1.9$ & $5.6 \pm 1.8$ & 0.04 \\
\hline Cellular mass, $\mathrm{kg}$ & $19.5 \pm 6.2$ & $17 \pm 5.7$ & 0.14 \\
\hline Cellular mass, $\%$ & $42.3 \pm 7.3$ & $35.9 \pm 7.8$ & 0.005 \\
\hline Total body muscle mass, kg & $22.6 \pm 4.9$ & $21.5 \pm 4.8$ & 0.43 \\
\hline \multicolumn{4}{|l|}{ Electrolytes } \\
\hline Sodium, mmol/L & $137.30 \pm 3.81$ & $136.36 \pm 4.52$ & 0.44 \\
\hline Potassium, mmol/L & $4.99 \pm 0.66$ & $4.72 \pm 0.65$ & 0.16 \\
\hline Phosphorus, mg/dL & $4.69 \pm 1.33$ & $4.74 \pm 1.10$ & 0.89 \\
\hline \multicolumn{4}{|l|}{ Functional assessment } \\
\hline Handgrip, kg & $27.1 \pm 10.0$ & $22.0 \pm 8.8$ & 0.07 \\
\hline ADL total score & $6 \pm 0$ & $5.8 \pm 1.1$ & 0.07 \\
\hline
\end{tabular}

ADL, activity of daily living; FFM, fat free mass

Bold values indicate statistically significant $P$ values. active and inactive patients showed significant correlation between total body muscle mass and handgrip values (active patients: $r=0.42 ; P=0.04$; inactive patients: $r=0.56 ; P=0.005$ ).

\section{Discussion}

Several studies indicated that sedentary behavior in patients on HD is associated with poor outcomes such as reduced survival and higher mortality $[3,18]$. The National Kidney Foundation recommends physical activity for patients on dialysis at least on most days of the week [19]. Barriers to physical activity play an important role in reducing the physical performance of this patient population. Similar to other studies $[16,19,20]$, in the present cross-sectional setting, the participation in physical activity was limited, as $51 \%$ of enrolled HD patients reported participating in light activity during leisure time never, almost never, or less than once per week, and $94 \%$ of patients reported facing barriers to physical activity.

Among our patient cohort, participation in physical activity was limited. Also, many reported barriers were significantly associated with physical inactivity, including reduced walking ability, too many medical problems, fear of getting hurt, not wanting to exercise, and feeling too old. This list of barriers suggests that physical inactivity is mainly due to a lack of motivation. In this regard, as previously indicated [16], promoting physical activity through tailored exercise interventions and psychological interventions might help to reduce barriers to physical activity in HD patients.

We found that active patients had higher intracellular water, cellular mass (\%), body mass cell index compared with inactive patients, indicating that active patients have an increased muscle compartment. It is likely that increased muscle mass was determined by a higher level of physical activity and that the selfreported level of physical activity, distinguished effectively active from inactive patients.

The use of body composition analysis appears clinically useful when identifying muscle mass derangement over time in inactive patients, although some limitations related to bioimpedance in HD patients should be considered [21]. However, this approach is not achievable in the majority of HD units because of the cost of the instrument and the lack of time to devote to this analysis and its interpretation by health care professionals. Therefore, the possible identification of a clinically useful biomarker of muscle efficiency would represent a simple and reproducible instrument to identify inactive patients.

Our data show that the myokine BAIBA, a small molecule secreted from myocytes [6], is reduced in HD patients compared with healthy and active controls, although no significant differences were observed between active and inactive patients, likely due to the reduced sample size. Interestingly, no differences between active and inactive patients were detected in terms of anemia, low Kt/v, or low albumin, which are markers often associated with the presence of asthenia.

A recent clinical trial demonstrated that plasma BAIBA concentrations increase with exercise and are inversely correlated with metabolic parameters, such as fasting glucose, insulin, homeostasis model assessment as an index of insulin resistance, triglycerides, and total cholesterol [6]. Thus, BAIBA may play a key role in exercise-induced protection from several metabolic diseases and it is identified as an exercise-triggered signal with relevant implications in the physiological mechanisms of physical exercise [22]. A previous study showed that BAIBA plasma concentrations are inversely correlated with cardiometabolic risk factors in humans and are increased during exercise training 
[6]. Moreover, it has been documented that BAIBA attenuates insulin resistance and improves glucose tolerance [17,23].

Moreover, we found a positive correlation between BAIBA plasma concentrations and high-density lipoprotein cholesterol, whereas we did not observe any association between statin treatment and BAIBA levels. Considering that statins often affect muscle cell metabolism [24], the possibility that they may alter BAIBA levels cannot be completely excluded. In adjusted analyses, we observed a correlation between BAIBA levels and the number and types of comorbidities. In particular, patients on HD with diabetes showed lower BAIBA levels than patients without diabetes, pointing out a crucial role of metabolic derangement, such as diabetes, in conditioning muscle performance.

Physical activity is considered an effective intervention for the treatment of insulin resistance and type 2 diabetes [25]. During exercise, secretion of BAIBA from skeletal muscle is increased, and BAIBA may contribute to exercise-induced protection from metabolic disease [6]. In experimental models, it has been revealed that BAIBA attenuates insulin resistance and inflammation induced by high-fat diet and prompts fatty acid oxidation in skeletal muscle, highlighting how the beneficial effects of exercise may be mediated through BAIBA [23].

Based on this evidence linking physical inactivity and BAIBA levels in HD patients, we found that BAIBA levels significantly correlated only with the two most frequent barriers endorsed by inactive patients. In fact, patients reporting both "fatigue on the dialysis days" and "reduced walking ability" showed lower BAIBA levels compared with those not reporting these two barriers. Therefore, we believe that these two conditions may be markers of quite important reduced physical activity, representing the two most common barriers in the present sample and in other larger cohorts $[15,16]$. On the other hand, no correlation between BAIBA and handgrip measurements has, to our knowledge, been documented. Also, no differences in handgrip values between active and inactive patients has been observed. Although handgrip measurements were found to be predictive of muscle mass in dialysis populations, this technique presents several limitations related to the choice of arm, patients' position, evaluation period, and diagnostic criterion [26].

A number of limitations of the present study remain. Our cohort of HD patients may not be representative of the entire dialysis population. The small sample size of the present cohort might have limited the possibility of recognizing associations between patient characteristics, especially inflammatory profile, and barriers to physical activity and/or BAIBA plasma levels. Additionally, the instrument used to assess physical activity during leisure time was not exhaustive and focused only on a subset of activities, not taking into consideration other activities that HD patients might perform. Increasing the number of enrolled patients and identifying other activities performed during leisure time might be a way to better understand how to improve participation in physical activity and to possibly use BAIBA as a targeted activity/inactivity biomarker in HD patients. The control group included only physically active, healthy individuals, and we did not assess the role of BAIBA in a more sedentary healthy group. We did not measure single nucleotide polymorphisms of alanine-glyoxylate aminotransferase 2 , which might affect BAIBA plasma levels, as recently shown in healthy volunteers [27]. We did not verify the kinetic of BAIBA before and after exercise, and we did not measure BAIBA levels after dialysis session. Moreover, a previous experience found a different trend in BAIBA levels between uremic patients and healthy individuals, although the methods used were different and with limited specificity [28].

\section{Conclusions}

Based on the findings of the present study, although BAIBA levels were not different when comparing active and inactive patients on HD, a relation in our cohort exists between the two most common perceived barriers to physical activity and BAIBA. A better understanding of the mechanism by which BAIBA influences metabolic and functional responses to exercise is therefore warranted in both experimental and clinical settings.

\section{References}

[1] Johansen KL, Delgado C, Bao Y, Tamura MK. Frailty and dialysis initiation. Semin Dial 2013;26:690-6.

[2] Muscaritoli M, Molfino A, Bollea MR, Rossi Fanelli F. Malnutrition and wasting in renal disease. Curr Opin Clin Nutr Metab Care 2009;12:378-83.

[3] Johansen KL, Kaysen GA, Dalrymple LS, Grimes BA, Glidden DV, Anand S, et al. Association of physical activity with survival among ambulatory patients on dialysis: the comprehensive dialysis study. Clin J Am Soc Nephrol 2013;8:248-53.

[4] Fiaccadori E, Sabatino A, Schito F, Angella F, Malagoli M, Tucci M, et al. Barriers to physical activity in chronic hemodialysis patients: a singlecenter pilot study in an Italian dialysis facility. Kidney Blood Press Res 2014;39:169-75.

[5] Johansen KL, Sakkas GK, Doyle J, Shubert T, Dudley RA. Exercise counseling practices among nephrologists caring for patients on dialysis. Am J Kidney Dis 2003:41:171-8.

[6] Roberts LD, Boström P, O'Sullivan JF, Schinzel RT, Lewis GD, Dejam A, et al Beta-aminoisobutyric acid induces browning of white fat and hepatic betaoxidation and is inversely correlated with cardiometabolic risk factors. Cell Metab 2014;19:96-108.

[7] Shimomura Y, Honda T, Shiraki M, Murakami T, Sato J, Kobayashi H, et al. Branched-chain amino acid catabolism in exercise and liver disease. J Nutr 2006;136(Suppl 1):250-3.

[8] Harper AE, Miller RH, Block KP. Branched-chain amino acid metabolism. Annu Rev Nutr 1984;4:409-54.

[9] Jankowska M, Debska-Slizien A, Rutkowski B. Bioelectrical impedance analysis before versus after a hemodialysis session in evaluation of nutritional status. J Ren Nutr 2006;16:137-40.

[10] Muscaritoli M, Molfino A, Chiappini MG, Laviano A, Ammann T, Spinsanti P, et al. Anorexia in hemodialysis patients: the possible role of des-acyl ghrelin. Am J Nephrol 2007;27:360-5.

[11] Molfino A, Don BR, Kaysen GA. Comparison of bioimpedance and dualenergy x-ray absorptiometry for measurement of fat mass in hemodialysis patients. Nephron Clin Pract 2012;122:127-33.

[12] Kaysen GA, Zhu F, Sarkar S, Heymsfield SB, Wong J, Kaitwatcharachai C et al. Estimation of total-body and limb muscle mass in hemodialysis patients by using multifrequency bioimpedance spectroscopy. Am J Clin Nutr 2005;82:988-95.

[13] Johansen KL, Dalrymple LS, Delgado C, Kaysen GA, Kornak J, Grimes B, et al. Association between body composition and frailty among prevalent hemodialysis patients: a US renal data system special study. J Am Soc Nephrol 2014;25:381-9.

[14] Tentori F, Elder SJ, Thumma J, Pisoni RL, Bommer J, Fissell RB, et al. Physical exercise among participants in the Dialysis Outcomes and Practice Patterns Study (DOPPS): correlates and associated outcomes. Nephrol Dial Transplant 2010;25:3050-62.

[15] Bossola M, Pellu V, Di Stasio E, Tazza L, Giungi S, Nebiolo PE. Self-reported physical activity in patients on chronic hemodialysis: correlates and barriers. Blood Purif 2014;38:24-9.

[16] Delgado C, Johansen KL. Barriers to exercise participation among dialysis patients. Nephrol Dial Transplant 2012;27:1152-7.

[17] Renal Data System. USRDS 2011 Annual Data Report. Bethesda, MD: National Institutes of Health, National Institute of Diabetes and Digestive and Kidney Diseases; 2011.

[18] Shlipak MG, Fried LF, Cushman M, Manolio TA, Peterson D, StehmanBreen C, et al. Cardiovascular mortality risk in chronic kidney disease: comparison of traditional and novel risk factors. JAMA 2005;293:1737-45.

[19] K/DOQI clinical practice guidelines for cardiovascular disease in dialysis patients. Am J Kidney Dis 2005;45(suppl 3):S1-153.

[20] Johansen KL, Chertow GM, Ng AV, Mulligan K, Carey S, Schoenfeld PY, et al. Physical activity levels in patients on hemodialysis and healthy sedentary controls. Kidney Int 2000;57:2564-70.

[21] Essig M, Vrtovsnik F. How to evaluate body composition in chronic kidney disease? Nephrol Ther 2008;4:92-8.

[22] Kammoun HL, Febbraio MA. Come on BAIBA light my fire. Cell Metab 2014;19:1-2. 
[23] Jung TW, Hwang HJ, Hong HC, Yoo HJ, Baik SH, Choi KM. BAIBA attenuates insulin resistance and inflammation induced by palmitate or a high fat diet via an AMPK-PPAR $\delta$-dependent pathway in mice. Diabetologia 2015;58:2096-105.

[24] Vrablik M, Zlatohlavek L, Stulc T, Adamkova V, Prusikova M, Schwarzova L, et al. Statin-associated myopathy: from genetic predisposition to clinical management. Physiol Res 2014;63:S327-34.

[25] Bogardus C, Ravussin E, Robbins DC, Wolfe RR, Horton ES, Sims EA. Effects of physical training and diet therapy on carbohydrate metabolism in patients with glucose intolerance and noninsulin-dependent diabetes mellitus. Diabetes 1984;33:311-8.
[26] Leal VO, Mafra D, Fouque D, Anjos LA. Use of handgrip strength in the assessment of the muscle function of chronic kidney disease patients on dialysis: a systematic review. Nephrol Dial Transplant 2011;26: 1354-60.

[27] Kittel A, Müller F, König J, Mieth M, Sticht H, Zolk O, et al. Alanine-glyoxylate aminotransferase 2 (AGXT2) polymorphisms have considerable impact on methylarginine and $\beta$-aminoisobutyrate metabolism in healthy volunteers. PLoS One 2014;9:e88544.

[28] Gejyo F, Kinoshita Y, Ikenaka T. Elevation of serum levels of betaaminoisobutyric acid in uremic patients and the toxicity of the amino acid. Clin Nephrol 1977;8:520-5. 